\title{
Numerical investigation of the aerodynamic breakup of Diesel droplets under various gas pressures
}

\author{
Dionisis Stefanitsis ${ }^{* 1,2,}$, Ilias Malgarinos ${ }^{1,2}$, George Strotos ${ }^{3}$, Nikolaos Nikolopoulos ${ }^{1}$, \\ Emmanouil Kakaras ${ }^{1}$, Manolis Gavaises ${ }^{2}$ \\ ${ }^{1}$ Centre for Research and Technology Hellas/Chemical Process and Energy Resources \\ Institute (CERTH/CPERI), Egialeias 52, Marousi, Greece \\ ${ }^{2}$ City University London, School of Engineering and Mathematical Sciences, Northampton \\ Square, EC1V OHB London, UK \\ ${ }^{3}$ Piraeus University of Applied Sciences, Mechanical Engineering Department, 250 Thivon \\ and P. Ralli str., Aegaleo 12244, Greece \\ *Corresponding author: stefanitsis@certh.gr
}

\begin{abstract}
The present study investigates numerically the aerodynamic breakup of Diesel droplets for a wide range of ambient pressures encountered in engineering applications relevant to oil burners and internal combustion engines. The numerical model solves the Navier-Stokes equations coupled with the Volume of Fluid (VOF) methodology utilized for capturing the interface between the liquid and the surrounding gas. An adaptive local grid refinement technique is used to increase the accuracy of the numerical results around the interface. The Weber (We) numbers examined are in the range of 14 to 279 which correspond to bag, multimode and sheet-thinning breakup regimes. Model results are initially compared against published experimental data and show a good agreement in predicting the drop deformation and the different breakup modes. The predicted breakup initiation times for all cases lie within the theoretical limits given by empirical correlations based on the We number. Following the model validation, the effect of density ratio on the breakup process is examined by varying the gas density (or equivalently the ambient pressure), while the We number is kept almost constant equal to 270; ambient gas pressure varies from 1 up to 146 bar and the corresponding density ratios $(\varepsilon)$ range from 700 down to 5 . Results indicate that the predicted breakup mode of sheet-thinning remains unchanged for changing the density ratio. Useful information about the instantaneous drag coefficient $\left(C_{d}\right)$ and surface area as functions of the selected non-dimensional time is given. It is shown that the density ratio is affecting the drag coefficient, in agreement with previous numerical studies.
\end{abstract}

\section{Keywords}

Droplet breakup, Diesel, VOF, density ratio, breakup initiation time.

\section{Introduction}

Droplet motion, deformation and breakup are observed in a wide variety of engineering applications such as in the injection systems of oil burners and internal combustion engines. Droplet deformation and subsequent breakup are caused by aerodynamic forces exerted on the drop by the surrounding gas, while surface tension and viscosity of the drop hinder deformation and tend to restore it to a spherical shape. The non-dimensional numbers that account for these effects are the Weber (We), Ohnesorge $(\mathrm{Oh})$ and Reynolds $(R e)$ numbers as well as the density $(\varepsilon)$ and viscosity $(N)$ ratios of the two phases [1]; the timescale used to non-dimensionalise the time is the shear breakup timescale [2]. For low Oh numbers $(O h<0.1)$ the droplet breakup is mainly controlled by the We number and according to [1] four different breakup regimes are observed as the We number increases. For We numbers in the range of 11 up to 35 the bag breakup mode is encountered during which the drop deforms into a bag resembling shape. As the We number is further increased up to 80 , the multimode regime starts to appear which is essentially a combination of the bag and sheet-thinning modes. In the sheet-thinning breakup mode $(80<W e<350)$ a sheet is formed at the periphery of the drop, which eventually breaks into ligaments. The final breakup mode is called catastrophic (We>350) and according to [1] is attributed to Rayleigh-Taylor and Kelvin-Helmholtz instabilities.

To the authors' best knowledge, a limited number of publications exist in the open literature regarding the experiments of droplet breakup utilizing Diesel as test fluid, which is the subject of the present work. The work of [3] investigated the breakup of Diesel droplets for We numbers ranging from 14 up to 10000 and Oh number equal to 0.027 . They produced photographic sequences of the breakup process and measured the breakup time and deformation. The series of works [4-6] have also examined Diesel fuel for conditions of $W e=56-532, R e=509-8088$, $O h=0.038-0.065$ and $\varepsilon=79-700$, corresponding to three breakup regimes, i.e. bag, sheet-thinning and catastrophic. 
They generated photographs of the breakup process and presented graphs for the drop deformation and trajectory. It was concluded that the breakup process in the three breakup regimes is independent of the $R e$ number and it is only a function of the We number for the range of $O h$ and $\varepsilon$ examined. The work of $[7,8]$ investigated the microscopic (droplet scale) and macroscopic (spray scale) breakup characteristics of Diesel droplets by introducing monodispersed droplets into a gas stream ejected from a nozzle. They examined We numbers from 4.3 up to 383 in three breakup regimes (vibrational, bag and sheet-thinning) and produced images of the breakup process and graphs for the droplet mean velocities and diameters.

Regarding the numerical modelling of droplet breakup, there are no studies (at least to the best of our knowledge) that investigate the breakup of Diesel fuel droplets corresponding to a range of We and Oh numbers, which are of practical interest. On the other hand, a few studies examine droplet breakup in Diesel engine conditions (i.e. low density ratios) without referring to specific fluid, while there are also some studies which examined the effect of density ratio on the breakup process. In [9] a front-tracking scheme in 2-D axisymmetric coordinates was used to study the breakup of impulsively accelerated drops. They investigated Diesel engine conditions (density ratios 1.15 and 10) and provided maps in the We-Re plane for the breakup outcome. They found that by increasing the $R e$ number the critical We number, that separates the different breakup regimes, decreases. The work of [10] used the Level-Set method in a 2-D axisymmetric domain to investigate the deformation of droplets at small Re numbers (25-200) and density ratios (2-32); they found that for density ratios above 32 the boundaries of the breakup regimes are almost unaffected by the density ratio. Furthermore, [11] simulated impulsively accelerated drops using a moving mesh interface tracking scheme and found that the drag coefficient is not affected much by the density ratio although it is larger than those of solid spheres at the same Re numbers. In [12] they used the Level Set method to simulate in a 2-D axisymmetric domain the deformation and breakup of liquid drops under the effect of gas flow. They performed three test simulations (free fall of droplet, bubble rising in liquid and droplet fall into a water pool) in order to validate their model and following this, they investigated the effect of the non-dimensional parameters (We, $R e, R e /$ and $\varepsilon$ ) on the breakup process. They concluded that the most influential parameter in droplet breakup phenomena is the We number followed by the $R e$ and $R e_{l}$, while the change in the density ratio from 10 to 100 led to alternation of the breakup mode. In [13] they studied droplet deformation and breakup on the bag and shear breakup regimes using a 3-D VOF method. They made parametric studies with different $W e, R e$ and $\varepsilon$ numbers and identified 5 new breakup modes in the multimode breakup regime. Moreover, they proposed a new breakup map in the $R e-N / \sqrt{\varepsilon}$ plane and concluded that any breakup regime can be represented in the proposed map, without any dependence on the We number. In the work of [14] they performed 3-D simulations of droplet breakup using a variant of the Coupled Level Set-VOF (CLSVOF) method called S-CLSVOF in the interFOAM solver of the OpenFOAM software package. They examined the sheet-thinning breakup regime under Diesel-like engine conditions and found that the density ratio affects the shape and size of the fragments and also the recirculation region of the gas flow.

To the author's best knowledge, the present work is the first one investigating the aerodynamic droplet breakup mechanism of Diesel fuel droplets under conditions of practical interest. The gas pressure and density are increased parametrically to cover a wide range of conditions, from atmospheric up to those corresponding to Diesel engine conditions. The following sections include initially a short description of the numerical model and the computational setup, followed by the CFD model validation against experimental data. After that, a parametric study is performed for the effect of density ratio on the breakup phenomenon while the most important conclusions are summarized at the end.

\section{Numerical model and computational setup}

The numerical model solves the Navier-Stokes equations coupled with the Volume of Fluid (VOF) [15] method for capturing the interface between liquid and gas. Surface tension forces are included in the momentum equation by using the Continuum Surface Stress (CSS) model of [16]. The CFD model has been validated and used for many applications including the aerodynamic breakup of droplets with high density ratios as described in [17-20].

The simulations are performed in a 2-D axisymmetric domain with the commercial CFD tool ANSYS FLUENT v16 [21], along with various user defined functions (UDFs) for the implementation of the adaptive local grid refinement [22] and the adaptive time-step for the implicit VOF solver. With the current grid resolution employed, drop radius is resolved by 192 cells per Radius ( $c p R)$ with minimum cell size ranging from $0.48 \mu \mathrm{m}$ up to $6.25 \mu \mathrm{m}$ depending on the droplet radius; systematic runs with $48,96,192$ and $384 \mathrm{cpR}$ have revealed that the resolution of $192 \mathrm{cpR}$ is adequate for achieving a grid independent solution.

\section{Model validation}

In order to evaluate the performance of the model the results are compared against the experimental data reported in the publications of [3-5]; in all these works, Diesel fuel was used as test fluid, along with the continuous air jet 
experimental setup. In [3] the droplets were introduced to the air jet with a syringe and a needle ensuring that they enter with almost zero initial velocity, while in [4] and [5] the droplets were introduced with a small initial velocity. The We number of interest in [3] is 14, which corresponds to the bag breakup regime and the droplet diameter is equal to $2.4 \mathrm{~mm}$. In [4] and [5] the examined We number ranges from 54 to 279, density ratios from 79 to 700 and drop diameters $184 \mu \mathrm{m}$ and $198 \mu \mathrm{m}$. The breakup modes observed in the experiments for these conditions were the bag and sheet-thinning.

The simulated cases for the model validation are presented in Table 1. Case 1 corresponds to the experimental study of [3], while cases 2-3 correspond to the experimental study of [4]. Cases 1-3 have a high density ratio equal to 695 ( $\left.P_{g}=1 \mathrm{bar}\right)$ and We numbers equal to 14,54 and 254 respectively. Case 4 corresponds to the experiment of [5] and have a small density ratio equal to $79\left(P_{g}=9.2 \mathrm{bar}\right)$ and We number equal to 264 . The Oh number for all cases is less than 0.1 so its effect on the phenomenon is considered to be insignificant [1].

Table 1: Validation cases.

\begin{tabular}{lllllllllll}
\hline Case & $P_{g}$ (bar) & $D_{0}(\mu \mathrm{m})$ & $U_{g}(\mathrm{~m} / \mathrm{s})$ & We & $R e$ & Oh & $\varepsilon$ & Breakup regime & $\begin{array}{l}\text { Reference } \\
\text { publication }\end{array}$ \\
\hline 1 & 1 & 2400 & 10 & 14 & 1540 & 0.027 & 695 & Bag & {$[3]$} \\
2 & 1 & 198 & 68 & 54 & 864 & 0.038 & 695 & Multi-bag & {$[4]$} \\
3 & 1 & 198 & 147 & 254 & 1867 & 0.038 & 695 & Sheet-thinning & {$[4]$} \\
4 & 9.2 & 184 & 52 & 264 & 5761 & 0.039 & 79 & Sheet-thinning & {$[5]$} \\
\hline
\end{tabular}

The temporal evolution of droplet shape and deformation for case 1 are presented in Figure 1 both for the simulation (denoted with the VOF iso-value 0.5) and the experiment. The droplet initially deforms into an ellipsoid shape $\left(t=1.4 t_{r e f}\right)$ which results in an increase of cross-stream deformation and a decrease in the streamwise one. Following that, a bag is formed ( $\left.t=1.75 t_{r e f}\right)$ and the deformation in both axes increases with the eventual breakup occurring at $t=1.85 t_{\text {ref. }}$ There is a good agreement between the simulation and the experiment as both predict bag breakup mode and a similar evolution for the droplet shape and deformation. The breakup initiation occurs earlier in the simulation at $t=1.85 t_{\text {ref }}$ instead of $2.93 t_{\text {ref }}$ in the experiment. Nevertheless, the predicted breakup initiation time of $t=1.85 t_{\text {ref }}$ is within the limits proposed in [23] and [24] (see Figure 5 of this section).

(a)

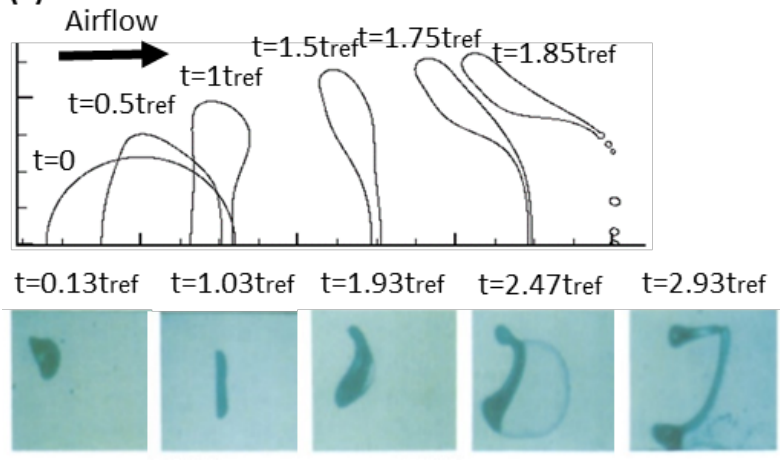

(b)

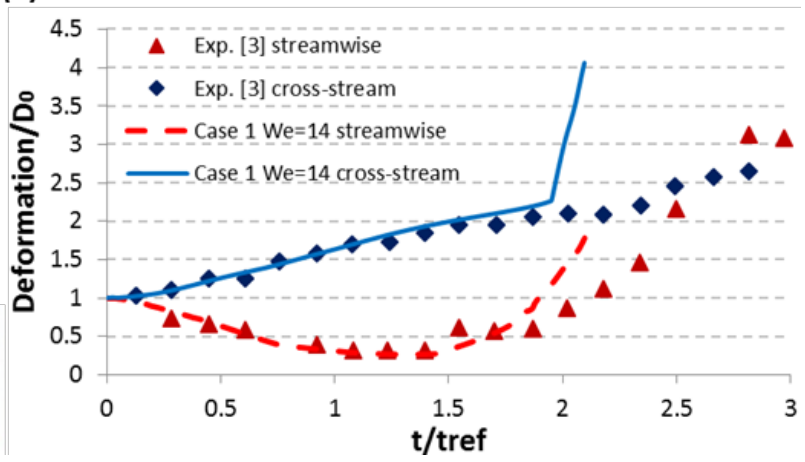

Figure 1. Temporal evolution of a) droplet shape and b) deformation from the simulation of case 1 and the experiment of [3].

The temporal evolution of droplet shape for cases 2-4 is shown in Figure 2. The sheet-thinning breakup mode predicted for cases 3 and 4 is in agreement with the experimental observations of [4] and [5] respectively. Nevertheless, for case 2, the present simulations predict a multi-bag (multimode) breakup mode characterized by a bag formed at the periphery of the droplet, while in the experiment of [4] a bag breakup was reported. The predicted multimode breakup for the examined range of We and Oh numbers is in accordance with the results reported in [25] and [1], which both report multimode breakup for the conditions of case 2. It should be mentioned that case 2 has been also simulated in a 3-D domain (additional to the 2-D axisymmetric) and the qualitative performance of this case, remained unchanged; the results of the 3-D simulation are not presented in this work. 


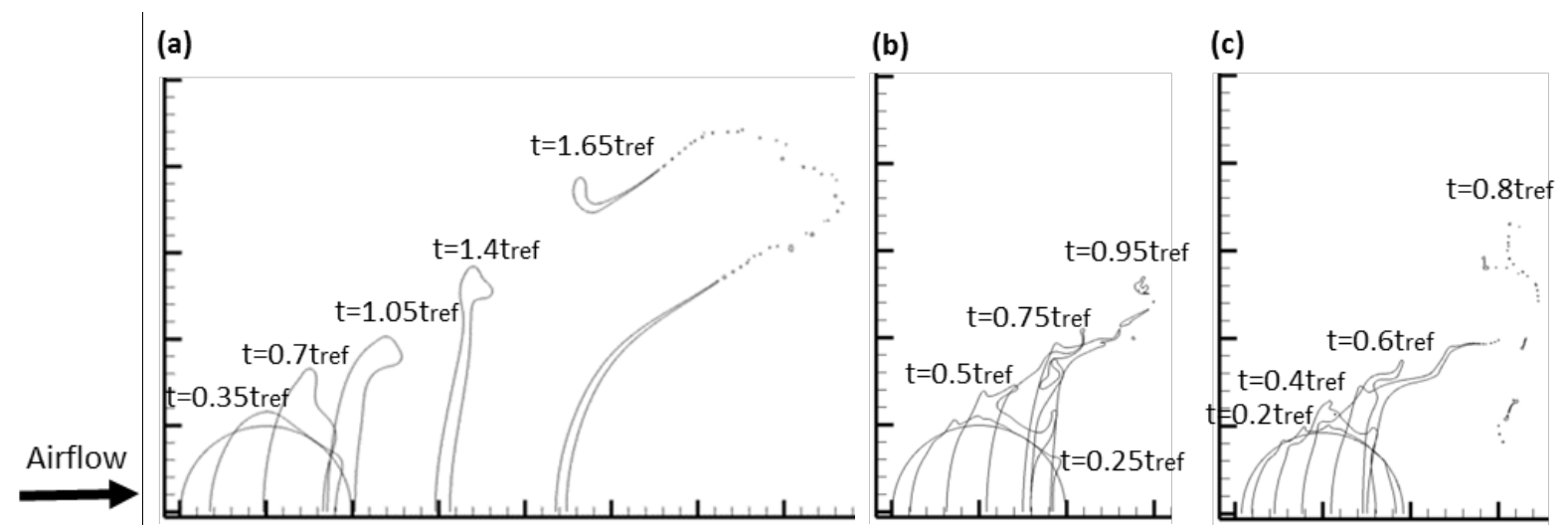

Figure 2. Temporal evolution of droplet shape for the cases 2, 3 and 4 (shown in a, b and c respectively).

Turning now to the quantitative comparison between the simulations and the experiments, the experimental measurements of [4] for the droplet deformation are initially provided as a function of the distance travelled along the direction of the cross-stream droplet motion (the reader is referred to the relevant paper [4] for details on the experimental setup). In the present 2-D axisymmetric approach, this secondary motion has been neglected. On the other hand, a comparison can still be made based on the corresponding experimental time needed for the droplet to travel along the cross-stream direction. This is estimated as $t=$ (cross-stream distance)/ $U_{d, 0}$; it has been assumed that the cross-stream velocity remains unchanged. The temporal variation of the droplet cross-stream deformation is presented in Figure 3, for cases 2 and 3. As it can be seen, there is a very good agreement between the results of the simulation and those of the experiment. Moreover, it is observed that the temporal variation of the deformation for the two cases is similar, although the We numbers differ significantly (54 compared to 254). This reveals the dimensionless character of the phenomenon when using the appropriate timescale.

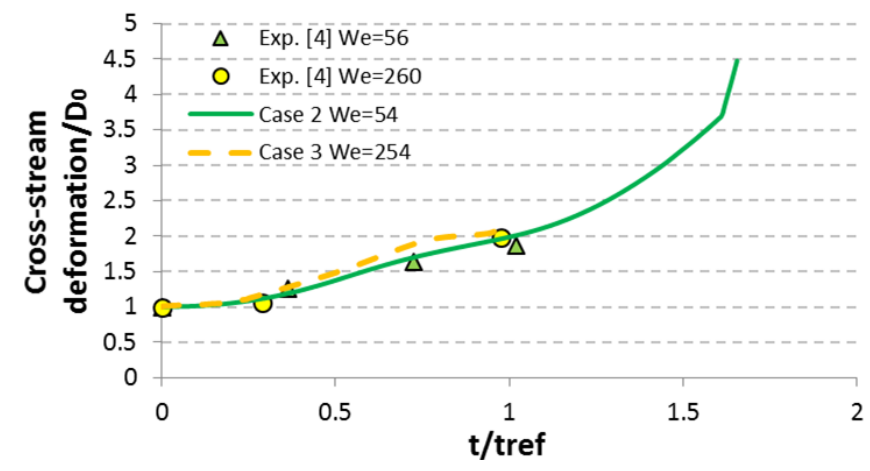

Figure 3. Drop deformation as function of non-dimensional time (cases 2-3 and experiment).

The temporal evolution of the non-dimensional droplet velocity $\left(U_{d} /\left(U_{g}-U_{d}\right)\right)$ versus a modified time $\left(\boldsymbol{t} /\left(\boldsymbol{t}_{\boldsymbol{r} e f} \sqrt{\boldsymbol{\varepsilon}}\right)\right)$ for all cases of Table 1 are compared against the experimental data of [24] in Figure 4; the axes have been nondimensionalised with the same fashion as in [24]. The dimensionless character of droplet velocity is confirmed for all cases; only case 4 shows a small deviation from the experimental data but it can be attributed to its low density ratio and high $R e$ number, compared to those examined in the experimental data. 


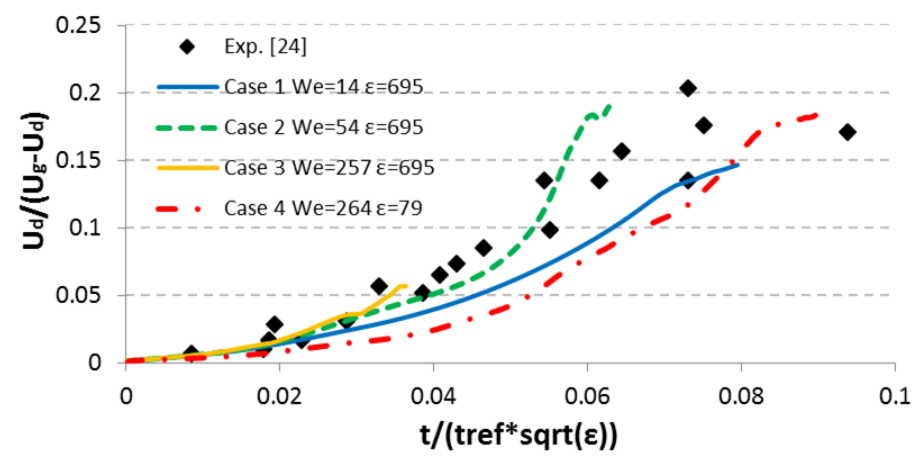

Figure 4. Non-dimensional droplet velocity as function of modified time for cases 1-4 and the experiment of [24]

The predicted breakup initiation time as a function of the We number is presented in Figure 5 for all cases of Table 1 along with the correlations proposed in [23] and [24]. In all cases, the breakup initiation time lies within the proposed limits and decreases with increasing We number. Cases 1-2 (bag and multi-bag breakup modes) give breakup initiation times closer to the correlation of [24], while cases 3-4 are closer to the correlation of [23].

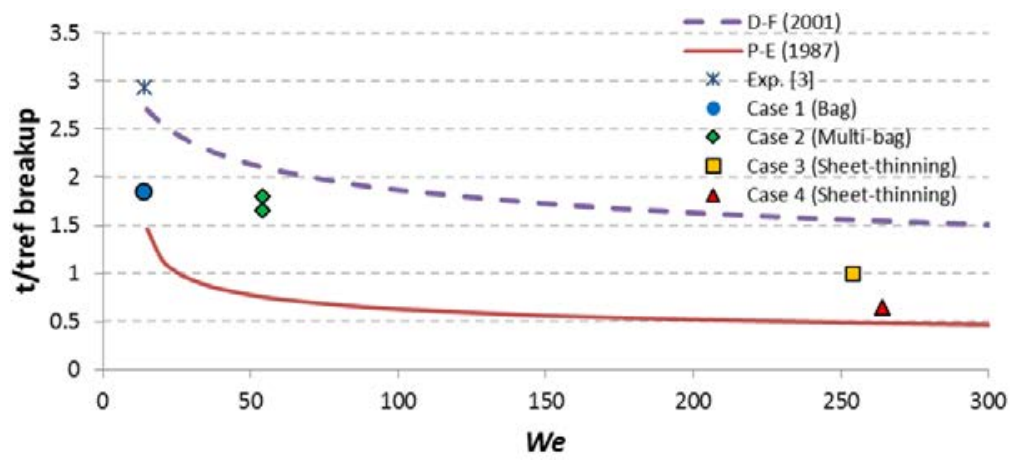

Figure 5. Breakup initiation time as function of We number (simulation and predictions in [23] and [24]).

\section{Parametric study}

Following the model validation, the numerical model is utilized to study the effect of density ratio (or equivalently the gas pressure) on the sheet-thinning breakup mode, for We numbers in the range of 265-279. The Oh number is constant and equal to 0.039 while the density ratio varies from 5 up to 700 (gas pressure varies from 146 down to 1 bar). The cases examined are presented in Table 2. Cases 5 and 6 have the smallest density ratios equal to 5 and 10 respectively. Case 8 is identical to case 4 of the validation part and cases 9-11 correspond to the experimental conditions of [5] (not presented in the validation part since case 4, with the lowest density ratio of 79 , is sufficient for validation purposes).

Table 2. Cases of parametric study.

\begin{tabular}{llllllll}
\hline Case & $P_{g}(\mathrm{bar})$ & $D_{0}(\mu \mathrm{m})$ & $U_{g}(\mathrm{~m} / \mathrm{s})$ & $W e$ & $R e$ & $\varepsilon$ & $\begin{array}{l}\text { Reference } \\
\text { publication }\end{array}$ \\
\hline 5 & 146 & 184 & 13.2 & 270 & 21503 & 5 & - \\
6 & 73 & 184 & 18.7 & 270 & 15205 & 10 & - \\
7 & 24 & 184 & 32.4 & 270 & 9297 & 30 & - \\
8 & 9.2 & 184 & 52 & 264 & 5761 & 79 & {$[5]$} \\
9 & 6.4 & 184 & 62 & 265 & 4829 & $112[5]$ \\
10 & 3.7 & 184 & 82 & 266 & 3688 & $195[5]$ \\
11 & 1 & 184 & 159 & 279 & 1920 & $700[5]$ \\
\hline
\end{tabular}

Starting from the breakup initiation time, this quantity slightly increases with the density ratio, being in the range of $0.45 t_{\text {ref }}$ to $0.70 t_{\text {ref. }}$ Regarding droplet deformation, the temporal evolution of liquid surface area (instead of the crossstream deformation presented so far) for all parametric cases is presented in Figure 6; this quantity is difficult to be measured experimentally and plays a very important role in combustion systems. After a short non-deforming period 
(0.25tref), the liquid surface area starts to increase. Up to the point of breakup initiation the liquid surface is not affected much by the density ratio while after that point a small deviation appears; nevertheless, the 2-D axisymmetric solution is not reliable after the breakup initiation, since 3-D effects become important.

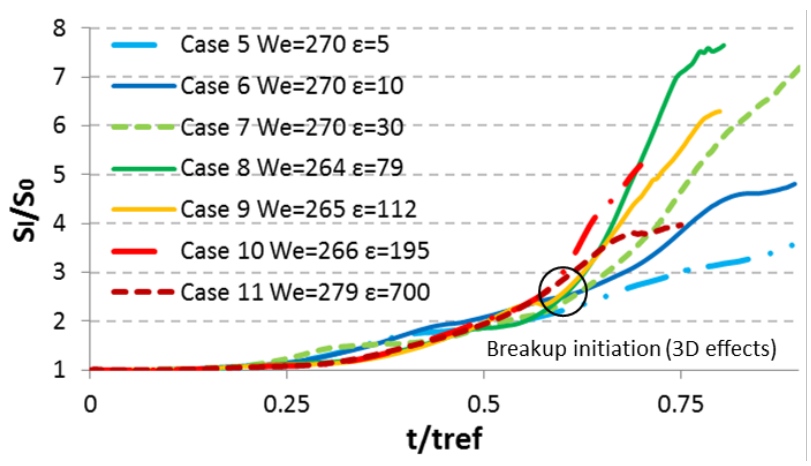

Figure 6. Liquid surface area as function of non-dimensional time (cases 5-11).

Another important parameter especially for spray codes, based on Lagrangian approaches, is the drag coefficient $\left(C_{d}\right)$. In order to calculate the instantaneous drag coefficient, the momentum equation for the droplet motion is employed:

$$
C_{d}(t)=\frac{\frac{4}{3} D_{0} \varepsilon \frac{d U_{l}(t)}{d t} \frac{A_{f}(0)}{A_{f}(t)}}{\left(U_{g}-U_{l}(t)\right)^{2}}
$$

The temporal evolution of drag coefficient is presented in Figure 7 a for selected cases. These are: case 6 with $\varepsilon=10$ (representative case for low density ratios), case 9 with $\varepsilon=112$ (representative case for moderate density ratios) and case 11 with $\varepsilon=700$ (representative case for high density ratios). The drag coefficient starts from a value close to 0.4 , which is the drag coefficient of solid spheres with Re number in the range 2000-20000 [26], and increases with time reaching values close to 1.17 , which is the drag coefficient of solid disks with Re number greater than 100 [26]. In addition, it is observed that the overall drag coefficient increases with decreasing density ratio. This trend can be explained by examining each term of eq. (1) separately: $D_{0}$ is the same for all cases, $A_{f, 0} / A_{f}$ is almost constant in all cases due to similar droplet shape and the term $U_{d}$ is small compared to $U_{g}$ for high We numbers so it can be ignored. Therefore, the ratio $\varepsilon / U_{g}=\rho /\left(\rho_{g} U_{g}^{2}\right)$ appearing in the equation, which is constant for constant We number, results in making the term $\mathrm{d} U_{d}(t) / d t$ for droplet acceleration the one that is the most influential among all, when changing the density ratio. The droplet acceleration is larger for lower density ratios (relatively lighter drops accelerate faster) thus making the drag coefficient higher as well. This is in agreement with the numerical study of [14] in which they state that generally, a lower density ratio results in a larger drag coefficient.

Due to the short duration of the phenomenon, it is interesting to examine the time-averaged drag coefficient for each case (calculated as the area under the curve of Figure 7a divided by the breakup time). This is shown as a function of the Re number in Figure $7 \mathrm{~b}$. The drag coefficient for all cases lies within the one of disk (purple dotted line) and the one of sphere (blue line) as taken from [26]. Again, we notice the same trend for the drag coefficient which increases with decreasing density ratio.

(a)

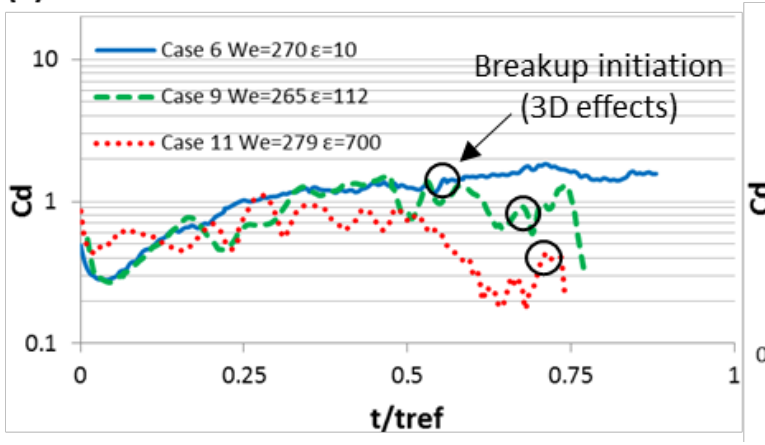

(b)

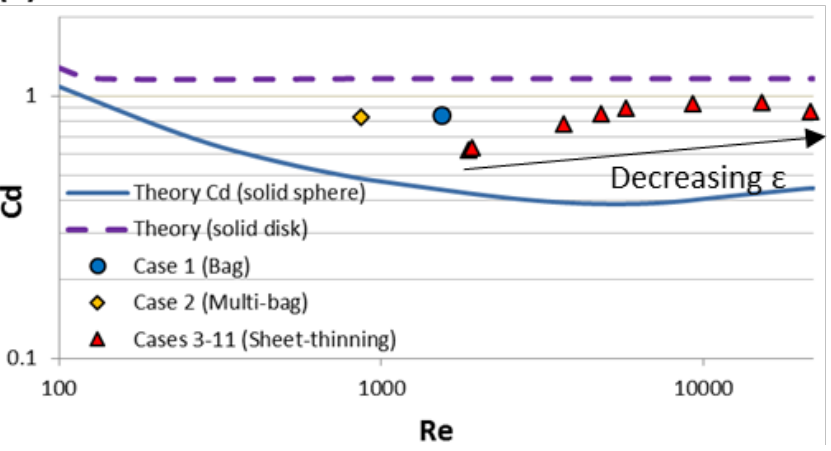

Figure 7. a) Temporal evolution of drag coefficient and b) time-averaged drag coefficient as function of $\operatorname{Re}$ (cases 1-11). 
Finally, a closer inspection of the breakup initiation time, reveals that decreasing the density ratio results in a decrease of the breakup initiation time (35\% for the lower density ratio examined). To account for this effect, the breakup initiation time proposed in [19] can be multiplied by the factor $1 /\left(1+\varepsilon^{-0.5}\right)$ which approaches unity for large density ratios.

\section{Conclusions}

In the present study the aerodynamic breakup of Diesel droplets under various density ratios was numerically investigated. The CFD model was initially validated against published experimental data for Diesel fuel and conditions of We numbers from 14 up to 264 and density ratios from 79 up to 695 . The predicted breakup regimes of bag and sheet-thinning as well as the temporal evolution of drop deformation are in close agreement with the experimental data. In one case the model predicts multi-bag breakup regime instead of bag as suggested by the experiments, but this is in accordance with the relevant experimental data. The dimensionless character of droplet velocity was confirmed for all the validation cases and also the breakup initiation times lie within the limits given by the experiments.

The parametric study performed for the density ratio (or equivalently the gas pressure) in the range of 5-700 has shown that the breakup regime and the temporal variation of the surface area of drop remain unaffected by the change in density ratio. On the other hand, the drag coefficient showed a decreasing trend with increasing density ratio in agreement with previous numerical studies. Finally, a correction factor has been proposed for the equation of breakup initiation time that accounts for the effect of density ratio.

\section{Acknowledgements}

Financial support from the MSCA-ITN-ETN of the European Union's H2O20 programme, under REA grant agreement $n .675676$ is acknowledged.

\begin{tabular}{|c|c|c|c|}
\hline \multicolumn{4}{|c|}{ Nomenclature } \\
\hline \multicolumn{2}{|c|}{ Roman symbols } & $U$ & Velocity [m/s] \\
\hline$A$ & Area $\left[\mathrm{m}^{2}\right]$ & We & Weber number [-] $W e=\rho_{g} U_{r e l, 0}^{2} D_{0} / \sigma$ \\
\hline$C_{d}$ & Drag coefficient [-] & \multicolumn{2}{|c|}{ Greek symbols } \\
\hline$D$ & Droplet diameter [m] & $\varepsilon$ & Density ratio $[-] \varepsilon=\rho_{l} / \rho_{g}$ \\
\hline$N$ & Viscosity ratio [-] $N=\mu_{l} / \mu_{g}$ & $\mu$ & Dynamic viscosity $[\mathrm{kg} /(\mathrm{s} \cdot \mathrm{m})]$ \\
\hline$P$ & Pressure [bar] & $\rho$ & Density $\left[\mathrm{kg} / \mathrm{m}^{3}\right]$ \\
\hline $\mathrm{Oh}$ & Ohnesorge number [-] $O h=\mu_{l} \sqrt{\rho_{l} \sigma \mathrm{D}_{0}}$ & \multicolumn{2}{|c|}{ Subscripts } \\
\hline $\operatorname{Re}$ & Reynolds number [-] $R e=\rho_{g} U_{r e l, 0} D_{0} / \mu_{g}$ & 0 & Initial \\
\hline$R e_{l}$ & Reynolds number for liquid [-] $R e_{l}=\rho_{l} U_{r e l, 0} D_{0} / \mu_{l}$ & $f$ & Frontal \\
\hline$S$ & Surface $\left[\mathrm{m}^{2}\right]$ & $g$ & Gas phase \\
\hline$t$ & Time $[\mathrm{s}]$ & I & Liquid phase \\
\hline$t_{\text {ref }}$ & Shear breakup timescale $[\mathrm{s}] t_{r e f}=\left(\mathrm{D}_{0} / U_{g, 0}\right) \sqrt{\varepsilon}$ & rel & Relative \\
\hline
\end{tabular}

\section{References}

[1] Guildenbecher, D. R., López-Rivera, C., and Sojka, P. E., 2009, "Secondary atomization," Experiments in Fluids, 46(3), pp. 371-402.

[2] Nicholls, J. A., and Ranger, A. A., 1969, "Aerodynamic shattering of liquid drops," AIAA Journal, 7(2), pp. 285290.

[3] Arcoumanis, C., Khezzar, L., Whitelaw, D. S., and Warren, B. C. H., 1994, "Breakup of Newtonian and nonNewtonian fluids in air jets," Experiments in Fluids, 17(6), pp. 405-414.

[4] Liu, Z., and Reitz, R. D., 1997, "An analysis of the distortion and breakup mechanisms of high speed liquid drops," International Journal of Multiphase Flow, 23(4), pp. 631-650.

[5] Lee, C. H., and Reitz, R. D., 2000, "An experimental study of the effect of gas density on the distortion and breakup mechanism of drops in high speed gas stream," International Journal of Multiphase Flow, 26(2), pp. 229244.

[6] Lee, C. S., and Reitz, R. D., 2001, "EFFECT OF LIQUID PROPERTIES ON THE BREAKUP MECHANISM OF HIGH-SPEED LIQUID DROPS," 11(1), pp. 1-19.

[7] Park, S. W., Kim, S., and Lee, C. S., 2006, "Breakup and atomization characteristics of mono-dispersed diesel droplets in a cross-flow air stream," International Journal of Multiphase Flow, 32(7), pp. 807-822.

[8] Kim, S., Hwang, J. W., and Lee, C. S., 2010, "Experiments and modeling on droplet motion and atomization of diesel and bio-diesel fuels in a cross-flowed air stream," International Journal of Heat and Fluid Flow, 31(4), pp. 667-679. 
[9] Han, J., and Tryggvason, G., 2001, "Secondary breakup of axisymmetric liquid drops. II. Impulsive acceleration," Physics of Fluids, 13(6), pp. 1554-1565.

[10] Aalburg, C., 2002, Deformation and breakup of round drops and nonturbulent liquid jets in uniform crossflows.

[11] Quan, S., and Schmidt, D. P., 2006, "Direct numerical study of a liquid droplet impulsively accelerated by gaseous flow," Physics of Fluids (1994-present), 18(10), p. 102103.

[12] Jing, L., and Xu, X., 2010, "Direct Numerical Simulation of Secondary Breakup of Liquid Drops," Chinese Journal of Aeronautics, 23(2), pp. 153-161.

[13] Kékesi, T., Amberg, G., and Prahl Wittberg, L., 2014, "Drop deformation and breakup," International Journal of Multiphase Flow, 66, pp. 1-10.

[14] Yang, W., Jia, M., Sun, K., and Wang, T., 2016, "Influence of density ratio on the secondary atomization of liquid droplets under highly unstable conditions," Fuel, 174, pp. 25-35.

[15] Hirt, C. W., and Nichols, B. D., 1981, "Volume of fluid (VOF) method for the dynamics of free boundaries," Journal of Computational Physics, 39(1), pp. 201-225.

[16] Lafaurie, B., Nardone, C., Scardovelli, R., Zaleski, S., and Zanetti, G., 1994, "Modelling Merging and Fragmentation in Multiphase Flows with SURFER," Journal of Computational Physics, 113(1), pp. 134-147.

[17] G. Strotos, I. M., N. Nikolopoulos, K. Papadopoulos, A. Theodorakakos, M.Gavaises, 2015, "Performance of VOF methodology in predicting the deformation and breakup of impulsively accelerated droplets," ICLASS 2015, 13th Triennial International Conference on Liquid Atomization and Spray Systems, August 23-27Tainan, Taiwan.

[18] Strotos, G., Malgarinos, I., Nikolopoulos, N., and Gavaises, M., 2016, "Predicting droplet deformation and breakup for moderate Weber numbers," International Journal of Multiphase Flow, 85, pp. 96-109.

[19] Strotos, G., Malgarinos, I., Nikolopoulos, N., and Gavaises, M., 2016, "Aerodynamic breakup of an n-decane droplet in a high temperature gas environment," Fuel, 185, pp. 370-380.

[20] Strotos, G., Malgarinos, I., Nikolopoulos, N., and Gavaises, M., 2016, "Numerical investigation of aerodynamic droplet breakup in a high temperature gas environment," Fuel, 181, pp. 450-462.

[21] "ANSYS®FLUENT, Release 16.0.."

[22] Malgarinos, I., Nikolopoulos, N., and Gavaises, M., 2015, "Coupling a local adaptive grid refinement technique with an interface sharpening scheme for the simulation of two-phase flow and free-surface flows using VOF methodology," Journal of Computational Physics, 300, pp. 732-753.

[23] Pilch, M., and Erdman, C. A., 1987, "Use of breakup time data and velocity history data to predict the maximum size of stable fragments for acceleration-induced breakup of a liquid drop," International Journal of Multiphase Flow, 13(6), pp. 741-757.

[24] Dai, Z., and Faeth, G. M., 2001, "Temporal properties of secondary drop breakup in the multimode breakup regime," International Journal of Multiphase Flow, 27(2), pp. 217-236.

[25] Hsiang, L. P., and Faeth, G. M., 1995, "Drop deformation and breakup due to shock wave and steady disturbances," International Journal of Multiphase Flow, 21(4), pp. 545-560.

[26] Clift, R., Grace, J. R., and Weber, M. E., 2005, Bubbles, drops, and particles, Courier Corporation. 